TP Periodica Polytechnica Electrical Engineering and Computer Science

59(3), pp. 118-124, 2015

DOI: $10.3311 /$ PPee. 8578

Creative Commons Attribution (i)

RESEARCH ARTICLE

\section{Multi-Agent Based Control Scheme for Electrical Distribution System Restoration}

\author{
A. Abdel Hafez ${ }^{1 *}$, Walid A. Omran ${ }^{1}$, Yasser G. Hegazy²
}

Received 10 May 2015; accepted after revision 21 August 2015

\begin{abstract}
Continuity of the electric supply is considered one of the main power quality indices, and thus, it is essential to perform fast and efficient restoration of electricity following outages. However, this might not be always an easy task, especially in large distribution networks. In this paper, an efficient multi-agent based control scheme is introduced to restore the maximum possible out of service loads without violating any of the technical constraints of the electric network. The proposed scheme utilizes a number of agents distributed along the feeder to gather the necessary information and communicate it to the decision making agent at the faulted feeder head. The decision making agent first attempts to restore all the out of service loads through group restoration. However, if this is not possible due to violating any of the technical constrains, then the agent attempts to restore as much loads as possible through a zone restoration process. To validate the effectiveness of the proposed control scheme, two case studies are presented to show the group and zone restorations. The distribution system is simulated in the MATLAB environment to perform the required power flow calculations and provide the necessary information to the agents. On the other hand, the agents are implemented in JADE environment where the communication and decision making process occurs.
\end{abstract}

\section{Keywords}

Distribution network, multi-agent system, restoration

\footnotetext{
${ }^{1}$ Faculty of Engineering, Ain Shams University, Egypt

${ }^{2}$ Faculty of Information and Engineering Technology, GUC, Egypt

*Corresponding author, e-mail: ahmedhafez2011@yahoo.com
}

\section{Introduction}

Restoration of electricity following an outage in the distribution network requires fast and effective action from the utility in order to minimize the impacts on the affected customers. The electrical power service restoration can be defined as finding an appropriate healthy feeder to transfer the out of service loads of a faulted feeder through a number of switching operations [1].

There are two main types of control strategies for autonomous restoration in a distribution network; Centralized Coordination Strategy (CCS) and Decentralized Coordination Strategy (DCS). The CCS depends mainly on one central control unit which collects all data from different locations along the feeder and takes the appropriate restoration decision. The control algorithm can be based on mathematical programming [2] or artificial intelligent techniques [3] to solve the restoration problem. The main advantage of such methods is their ability to obtain an optimal restoration path. However, they require extensive calculations and long processing time, especially in case of large networks [4].

On the other hand, the DCS utilizes distributed controllers for data collection while the decision making is achieved by a specific controller which is assigned based on the condition of the system. Such methods are usually based on an expert system or heuristic techniques for solving the restoration problem $[5,6]$,which are usually fast and less computationally extensive. Moreover, they are not dependent on a single central unit, and thus, they enhance the reliability of the control system. In [7], a combination of heuristic and expert based system is introduced for service restoration after fault isolation using a SCADA system for information collection and decision making. However, the method has a central unit for decision making for all cases which highly reduces the reliability of the control system. Recently, the use of Multi-Agent System (MAS) in the restoration process is proposed by several researchers. For example, in [8] a multi-agent system is proposed for intelligent restoration in the distribution network using one type of agents having two operating modes. The method takes into consideration the current limits of the feeders but does not consider the voltage limits for the restoration path which might lead 
to an unacceptable operating condition after restoration. The work in [9] introduces a self-healing mechanism in distribution systems using a multi-agent framework using an expert based algorithm. The system consists of two types of agents forming two layers where the higher one is responsible for the decision making. The algorithm considers the current and voltage limits for the healthy feeders but neglects the voltage limits in the restored feeder which might lead to unacceptable operating conditions in the restored part.

In this paper, an approach based on MAS is implemented for isolating the faulted part of the feeder and solving the restoration problem. The approach takes into consideration the current and voltage limits in both the healthy and the restored sections of the network. The MAS consists of a number of agents distributed along the network to gather the necessary information while the decision making agent is defined in case of fault as the agent at the head of the faulted feeder.

\section{Expert Based Restoration Problem Formulation}

The restoration problem can be formulated as a large scale combinational problem due to the many combinations of possible switching operations, especially in large distribution networks $[8,10]$. One possible and effective method to solve such problems is to use a well-designed expert based algorithm that attempts to achieve the required objectives while respecting the technical constraints of the system. The main objectives of the restoration problem are: (1) attempt to maximize the restored out of service loads to increase the quality and reliability of the supply, and, (2) attempt to minimize the switching operations to increase the lifetime of the switches and reduce the cost of operation.

The problem is subject to several technical constraints that must be maintained during and after the restoration process to ensure an acceptable operation of the distribution system. These constraints can be summarized as follows:

1. Maintain the radial structure of all feeders

2. Maintain the current limit for all feeder branches

$$
I_{j} \leq I_{\max j}
$$

where $I_{j}$ is the magnitude of current through branch $j$, and $I_{\max }$ is the maximum allowable current for this branch

3. Maintain the voltage limit at all buses along the healthy feeder. This can be achieved by calculating the current limit corresponding to the voltage limit of the healthy feeder as in [11]:

$$
I_{v_{h}}=\frac{V_{b h}-V_{\min }}{Z_{p h}}
$$

where $I_{v_{h}}$ is the maximum additional current that can flow through the healthy feeder without violating its voltage limit, $V_{b h}$ is the voltage magnitude of bus $h$ having the lowest magnitude along healthy feeder, $V_{\text {min }}$ is the minimum allowable bus voltage magnitude (e.g., 0.9 p.u.), and $Z_{p h}$ is the magnitude of impedance for the portion of the healthy feeder contributing to the restoration path starting from the substation to the bus closest to bus $h[11]$.

4. Maintain the voltage limit at all buses of the restored part of the faulted feeder. This can be achieved by calculating the current limit corresponding to the voltage limit of the restored part by:

$$
I_{v_{f}}=\frac{V_{b t}-V_{\min }}{Z_{p f}}
$$

where $I_{v_{f}}$ is the maximum additional current that can flow through the restored feeder without violating its voltage limit, $V_{b t}$ is the magnitude of the tie bus voltage of the healthy feeder, $V_{\min }$ is the minimum allowable bus voltage (e.g., 0.9 p.u.), $Z_{p f}$ is the magnitude of impedance for portion of the total path of the restoration including the restored part and can be calculated as $Z_{p f}=\left|Z_{p t}+0,5 Z_{f}\right|$ where $Z_{p t}$ is theimpedance of the restoration path from the substation to the tie bus along the healthy feeder and $Z_{f}$ is the series impedance of the out of service zones considering the assumption that the out of service loads are uniformly distributed along the faulted feeder [12]. The zone is defined as a section of the feeder which is bound by two or more switches. Each zone has its own measuring devices which measure the incoming and outgoing currents.

The work done in this paper is based on the following assumptions:

1. The out of service loads are uniformly distributed along the faulted feeder to calculate the out of service loads that can be restored without violating the voltage constraint in the faulted section.

2. The power loss in the distribution feeder is not considered in the restoration process. This is because the main objective is to maximize the number of restored out of service loads regardless of the power loss as this is considered as an abnormal operating condition [13].

3. The bus voltage angles of the healthy feeder following the restoration are assumed to be close to the angles before the restoration $[9,12]$. This is due to the fact that the demand of the restored sections is usually small as compared to the demand of the healthy feeder.

\section{Proposed MAS Architecture}

The proposed MAS is implemented using a number of micro-controller based devices installed and distributed along the network, where this MAS proposed structure consists of three main types of agents: (1) Feeder Agent placed at each feeder head circuit breaker, (2) Tie Agent placed at each zone having a tie switch which is normally open during normal 
operation, and (3) Zone Agent placed at each zone along the feeder. The Feeder Agent of the faulted feeder (FFA) is responsible for the decision making of the restoration process as will be explained in the following section. The FFA identifies itself once the circuit breaker at the head of the faulted feeder trips. The Tie Agent for each healthy feeder which can be connected to the faulted feeder (HTA) is responsible for calculating the limits of its healthy feeder in case the power is restored through it and then sends the information to the FFA. The HTA identifies itself once it receives a Call for Proposal (CFP) message from the FFA. The Zone Agent can either be a Faulted Zone Agent (FZA), a Down Zone Agent (DZA) or a Healthy Zone Agent (HZA). The FZA is the agent of the zone that has the fault. The main function of this agent is to isolate the fault by opening the switches at the terminals of the zone once the circuit breaker is tripped. The FZA identifies itself once it detects a large difference between the incoming and outgoing currents as compared to the normal operating conditions. The DZA is the agent of the zone that has no power due to the isolation of the fault. This agent identifies itself once it receives a message from the FFA to send the demand power of its zone. The HZA is the agent of the zone along the restoration path of the healthy feeder. The main function of the HZA is to identify the minimum bus voltage within the zone and to calculate the available spare capacity of the branches within the zone that does not violate the current limit. This agent identifies itself once it receives a message from its HTA to send information about its zone for calculating the feeder limits.

The communication between the agents is done using an Agent Communication Language (ACL) which contains various types of messages such as inform, request and call for proposals [14]. This language was developed by the Foundation for Intelligent Physical Agents (FIPA), an IEEE standard organization, which specifies software standards for agent based systems [15].

\section{Proposed Restoration process}

The restoration process involves two stages, first, to detect the fault and isolate the faulted part of the feeder, and second, to initiate the restoration algorithm in order to attempt restoring the out of service loads.

0 algorithm to restore the out of service zones in the downstream of the faulted zone. In this stage, the algorithm attempts first to perform group restoration to restore all the out of service loads in the downstream part of the faulted feeder by switching them to another feeder through a single switching operation. If group restoration is not possible due to the violation of one or more of the technical constraints, then zone restoration is initiated. In this case, the algorithm attempts to restore as much zones as possible without violating any of the constraints. To perform the restoration process, the FFA communicates with the DZAs to gather information about the total demand power required for restoring the downstream zones. The FFA also communicates with the HTAs to get information about the power that they can supply without violating of any constraint of their healthy feeders. After gathering the required information, the FFA decides which is the most suitable path for restoration and sends its decision to the selected HTA to close its tie switch.

The role of different agents during the restoration process can be illustrated in the following sections.

\subsection{Faulted Feeder Agent (FFA)}

Once the fault is isolated and the upstream zones are restored, the FFA communicates with the DZAs and HTAs to collect the necessary information in order to take the decision about the restoration process. The sequential operation of the FFA is as follows:

1. FFA sends Request for Information (RFI) messages to all DZAs, to identify the needed demand power for each down zone.

2. DZAs respond to the FFA with inform message containing the needed demand power for restoration.

3. FFA sends CFP messages to all the available HTAs.

4. Each HTA responds to the FFA with an inform message containing the magnitude of the tie bus voltage of the healthy feeder and the Allowable Power for Restoration $(A P R)$ that will not violate the branch current limits and voltage limits in the healthy feeder as will be shown in Eq. (9).

5. After the information is collected by the FFA, the decision making algorithm is initiated to perform the restoration process according to the expert based rules as illustrated in Section 2. The FFA calculates $I_{v_{f}}$ using Eq. (3), and hence, the corresponding power is estimated by:

$$
A P R_{v f}=\left|V_{L}\right| *\left|I_{v f}\right|
$$

Where $\left|V_{L}\right|$ is assumed to be 1 p.u.

6. FFA calculates the power that can be restored for each tie after considering the voltage limits in the restored part as:

$$
A P R_{m_{i}}=\min \left(A P R_{i}, A P R_{v f_{i}}\right)
$$

where $A P R_{m_{i}}$ : is the allowable power for restoration from healthy feeder $i$ without violating the current or voltage limits for the healthy and the restored zones.

7. FFA compares the $A P R_{m_{i}}$ of all healthy feeders with the total demand of the down zones to check the possibility of performing group restoration based on the condition:

$$
\max _{i \in n_{h}}\left(A P R_{m_{i}}\right) \geq \sum_{j=1}^{n_{z}}\left|S_{j}\right|
$$

where $S_{i}$ is the demand power of the down zone $j, n_{z}$ is the total number of out of service zones, and $n_{h}$ is the total number of available HTAs.

8. FFA sends an accept proposal message to the HTA satisfying the conditions of group restoration. 
9. If Eq. (6) is not satisfied, the FFA initiates zone restoration by building up Zone/Switch relation table [17] to identify all possible combinations that can be done on the out of service zones for each HTA

$$
Z_{c}=\left\{Z_{c_{x}}=\bigcup_{j=1}^{n_{z}} \text { Zone }_{j}\right\}
$$

where $Z_{c}$ is the set of all possible zone combinations, $Z_{c_{x}}$ is zone combination number $x$ and is the total number of out of service zones.

10. FFA calculates the needed demand power by each combination of zones in order to be restored, and then calculates the new $A P R_{m_{i}}$ for each zone combination, but in this case $Z_{f}$ will be different for each combination.

11. FFA arranges the combinations in descending order according to the needed demand power and compares the needed demand power with the corresponding $A P R_{m_{i}}$ for each combination.

12. If a combination has the needed demand power less than or equal the $A P R_{m_{i}}$ from the $i^{\text {th }}$ tie, FFA sends an accept proposal message to HTA $i$, requesting to restore the power and to perform the necessary switching operation to reach this combination.

13. FFA sends request messages to the selected DZAs in order to reach the desired combination including the switch to be opened and switch to be closed, then sends accept proposal message to the selected HTA in order to close the tie switch.

14. FFA updates the zone switch table after eliminating the combinations that can't be used due to radial constraint as indicated in Section 2, and then if table is empty the restoration is done successfully.

15. If the table is not empty, repeat the steps from 11 to 14 till the table is empty or this is the last available HTA.

\subsection{Healthy Tie Agent (HTA)}

Once the Tie Agents receive CFP messages from the FFA they identify themselves as HTAs. Each HTA collects information about its feeder to identify how much power can be provided to the restored loads without violating any of constraints of the healthy feeder as illustrated in Section 2 (i.e. calculate the $A P R$ ). The HTA sends request messages to all zones along the restoration path, which define themselves as Healthy Zone Agents (HZAs) after receiving this message, requesting information to determine the maximum power it can support without violating the constraints of the healthy feeder. The sequential operation of the HTA is as follows:

1. HTA receives a CFP message from the FFA.

2. HTA sends RFI messages to the HZAs of its feeder, requesting the minimum bus voltage within the zone and spare capacity of each zone which can be calculated as:

$$
I_{\text {avaj }}=\min _{k}\left(I_{\max k-j}-I_{k-j}\right)
$$

Where $I_{a v a j}$ is the available spare current of zone $j$, $I_{\max k-j}$ is the maximum current carrying capacity for branch $k$ of zone $j$ along the restoration path, $I_{k-j}$ is the magnitude of actual current flowing in branch $k$ of zone $j$.

3. HTA calculates the voltage limit for the healthy feeder $I_{v_{h}}$ using Eq. (2) and calculates the available spare current that can be fed by the healthy feeder without violating the current constraint of its branches, $I_{c h}$, by:

$$
I_{c h}=\min _{i}\left(I_{\text {ava } i}\right)
$$

4. HTA calculates the $A P R$ by:

$$
\begin{gathered}
A P R=\left|V_{L}\right| *\left|I_{\text {spare }}\right| \\
I_{\text {spare }}=\min \left(I_{c h}, I_{v h}\right)
\end{gathered}
$$

where $\left|V_{L}\right|$ is assumed to be 1 p.u. and $I_{\text {spare }}$ is the available spare current that can be used for restoration taking into consideration the current and voltage constraints along the healthy feeder.

5. HTA sends an inform message to the FFA containing the $A P R$ and the tie bus voltage.

6. HTA waits for an accept proposal message from the FFA agent in order to close the tie switch and energize the downstream zones of the faulted feeder.

\subsection{Zone Agents}

As mentioned before, the zone agents are placed along the feeder and are divided into three types, each having a specific function.

The sequential operation of the FZA is as follows:

1. FZA identifies the faulted zone.

2. FZA sends a signal to the switches at the terminals of the zone to open and isolate the fault.

3. FZA sends a signal to the circuit breaker to reclose and supply power to the upstream zones of the faulted feeder.

For the DZA, the sequential operation is as follows:

1. DZA receives RFI message from FFA, requesting the needed demand of its zone.

2. DZA sends back an inform message containing the load demand of its zone.

3. If the DZA receives a request message from the FFA, it performs the required switching operation as indicated in the message.

Finally, the sequential operation of the HZA is as follows:

1. HZA receives RFI message from its HTA, requesting information about the limits of the zone.

2. HZA calculates the $I_{\text {ava }}$ using Eq. (8).

3. HZA replies with and inform message containing the 
available spare current of the zone and the minimum bus voltage within the zone.

\section{Simulation Cases}

In order to demonstrate the effectiveness of proposed MAS restoration process, two case studies are simulated using the data of the $11 \mathrm{kV}$ radial distribution system shown in Fig. 1 [18]. The system is modeled in the MATLAB environment to perform the required power flow calculations. On the other hand, the agents are implemented in Java Agent Developing Framework (JADE) [19]. The power flow calculations are carried out pre-fault to obtain the information that the MAS require in order to calculate the different constraints. The power flow calculations are also carried out after the restoration decision is taken by the MAS in order to validate the correctness of the decision.

\section{1) Case 1:}

A permanent fault is simulated insidezoneZa7of Feeder „a”. In this case, FCB-1 trips to stop feeding the fault, while the Zone Agent of Zone Za7 identifies itself as the FZA after it detects an abnormal condition. The FZA sends a signal to the switches of Zone Za7 to isolate the fault and then sends a signal to FCB-1 in order to reclose and energize the upstream part of the faulted feeder. The out of service zones are Za8 to Z10 and they need a total demand power 0.137 p.u. The available ties are Tie 2 at Feeder ,c" and Tie 3 at feeder ,d" with corresponding tie switches $\mathrm{S} 12$ and $\mathrm{S} 10$.

After the fault is isolated, the Feeder Agent located at FCB-1 of Feeder ,a" identifies itself as the FFA, and starts the restoration process as illustrated in Section 4. First, the FFA sends RFI messages to all DZAs (Za8, Za9 and Za10) to identify the needed demand power for each down zone. Each DZA responds with an inform message containing the load demand of its zone. The FFA then sends CFP messages to the HTAs at Tie 2 and Tie 3. Each HTA sends RFI to all their HZAs, requesting the minimum available spare capacity of its branches and its minimum bus voltage. Each HZA calculates it'sand sends inform message with spare capacity and its minimum bus voltage. The HTA calculates the $A P R$ using Eq. (9), where the $A P R$ for the HTA at Tie 2 is 0.265 p.u.and for the HTA at Tie 3 is 0.0888 p.u.. Then the HTA sends an inform message to the FFA containing the $A P R$ for each tie and the tie bus voltage (0.93088p.u. for Tie 2 and 0.9087p.u. for Tie 3). After receiving this information, the FFA calculates the $A P R_{m_{i}}$ for each healthy tie, where for Tie 2 it is 0.1898 p.u. and for Tie 3 it is 0.0604p.u. The FFA compares the highest $A P R_{m_{i}}$ with the needed demand power by DZAs using Eq. (6). In this case, the restoration can be done through group restoration. Thus, the FFA sends an accept proposal message to the HTA at Tie 2 in order to close switch S12 and restore all the down zones through one switching operation. After the restoration is done, the minimum bus voltage is 0.9055 p.u. at L64 along Feeder „c”, which is the new restored part with a value does not

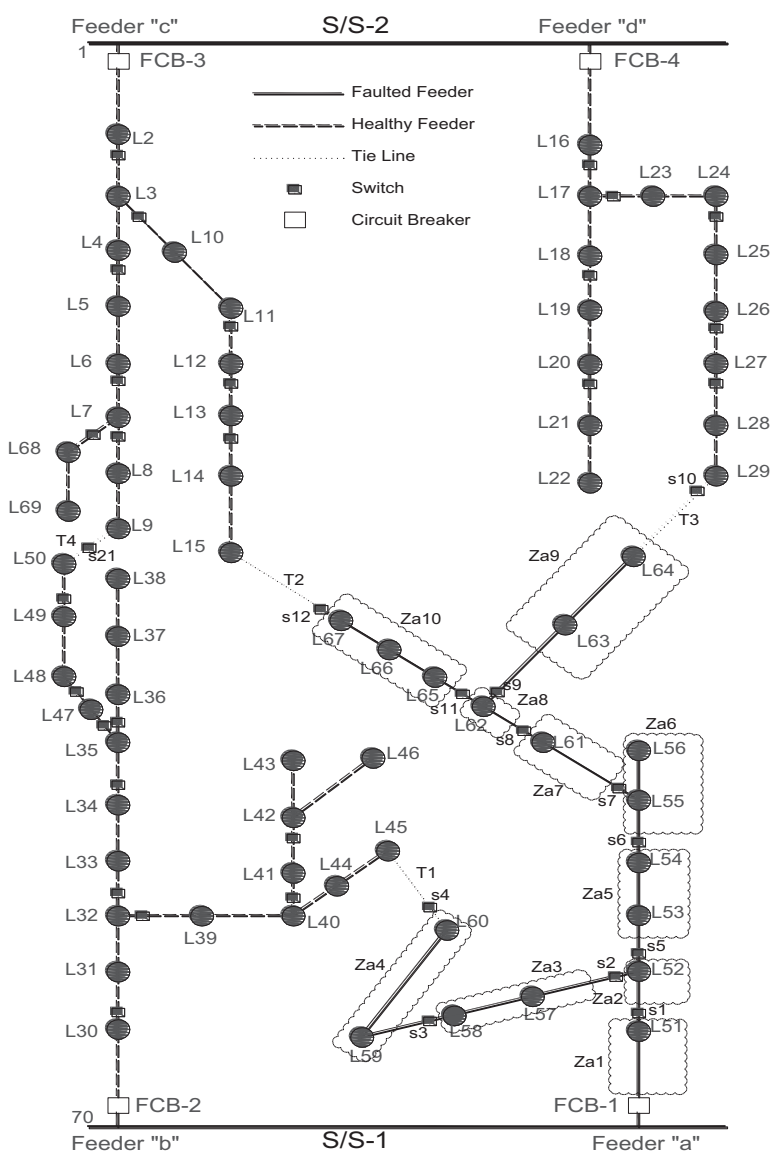

Fig. 1 Test distribution system

violate the voltage limit. Feeder ,d” remains unchanged because it didn't participate in the restoration process.

\section{2) Case 2:}

In this case, the fault is simulated in Zone Zal of Feeder ,a”. The detection and isolation of the fault is the same as Case 1, where the Zone Agent of Zone Za1 is the FZA. In this case the FZA does not send a signal to the circuit breaker to reclose because no zones can be restored after reclosure. Thus, the down zones are $\mathrm{Za} 2$ to $\mathrm{Z} 10$ and they need a total demand power 1.3716p.u. The available ties are Tie 1, Tie 2 and Tie 3, with corresponding tie switches S4, S12 and S10, respectively.

After the fault is isolated, the FFA sends RFI messages to all DZAs to identify the needed demand power for each DZA. The DZA respond with inform message containing the load demand. After that FFA send CFP messages to the HTAs which then send messages to their HZAs. After receiving the required information, each HTA calculates the $A P R$ for each tie. The FFA receives an inform message from the HTAs containing the $A P R$ and the tie voltage of each tie. These are 0.6779 p.u.and 0.953674 p.u. for Tie 1, 0.265p.u. and 0.93088p.u. for Tie 2, and, 0.0888p.u. and 0.9087p.u. for Tie 3, respectively. The FFA then calculates the $A P R_{m_{i}}$ for each tie, where for Tie 1 it is 0.362 p.u., for Tie 2 it is 0.147 p.u. and for Tie 3 it is 0.0454 p.u. The FFA compares the highest $A P R_{m_{i}}$ with the neededdemand power by all DZAs using Eq. (6). In this case, group restoration 


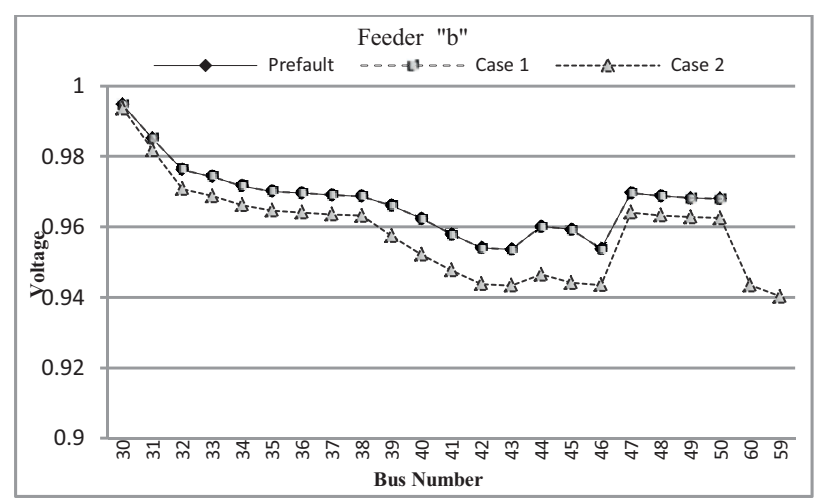

Fig. 2 Voltage profile of Feeder "b"

is not possible, thus, zone restoration is initiated. The FFA builds the zone switch relation table by Eq. (7) to identify all possible combinations to be restored through each tie. Then the FFA calculates the new $A P R_{m_{i}}$ for each zone combination, as $Z_{f}$ will be different for eachcombination.

The FFA then arranges the combinations in a descending order according to the needed demand power. It then compares the $A P R_{m_{i}}$ for each combination with the load demand of this combination.

After searching the combinations throughout the table, it is found that there are no possible combinations that can be restored from $\mathrm{T} 3$ to maintain the radial constrain. The possible combinations from Tie 1 and Tie 2 are identified to be:

1. Za4 from Tie 1 , as the needed demand power is 0.2321 p.u. and the $A P R_{m_{1}}$ for this combination is 0.6074 p.u.. Restoring $\mathrm{Za} 3$ is not possible as this combination needs a demand power of 0.6673 .

2. $\mathrm{Za} 7, \mathrm{Za} 8, \mathrm{Za} 9$, and $\mathrm{Za} 10$ from Tie 2, as the total needed demand power is 0.1365 p.u. and the $A P R_{m_{2}}$ for this combination is 0.1981 p.u.. Restoring Za6 is not possible as this combination needs a demand power of 0.2427 .

The first combination can be achieved by opening S3 and closing S4 while the second combination can be achieved by opening S7 and closing S12. Thus, the FFA sends request messages to the DZAs of Za4 and Za7 to open switchesS3 and S7, respectively. Then, the FFA sends accept proposal messages to Tie 1 and Tie 2 in order to close closing S4 and S12, respectively to restore the power. After the restoration is done, the minimum bus voltage along Feeder „„” is 0.94019 p.u. at L59, and along Feeder „c" is 0.90554 p.u. at L64 which does not violate the voltage limit.

For demonstration, the voltage profiles for the feeders involved in restoration process in the two case studies are presented in Fig. 2 and Fig. 3. The figures show that all the bus voltages are within the permissible limits.

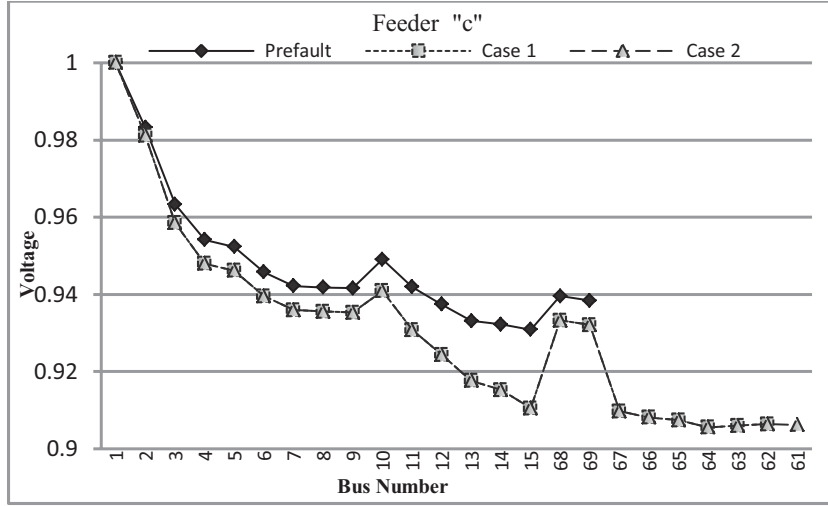

Fig. 3 Voltage profile of Feeder "c"

\section{Conclusion}

In this paper, a Multi-Agent System is used for electrical service restoration in distribution networks. The proposed system is composed of three types of agents installed according to their place in the distribution network. The system is based on an expert based algorithm to meet the restore as much loads as possible without exceeding the current carrying capacity of the branches or violating the voltage limits of the buses. For the sake of verifying the proposed control scheme two scenarios are presented to show the concept of group restoration and zone restoration. The simulation results show that the restoration can be achieved successfully using the proposed scheme which allows the network to have an efficient self-healing capability that could be enrolled in smart grid distribution systems.

\section{References}

[1] Chen, W-H. "Quantitative decision-making model for distribution system restoration." IEEE Transactios on Power Systems. 25 (1). pp. 313 321. 2010. DOI: 10.1109/tpwrs.2009.2036811

[2] Nagata, T., Sasaki, H., Yokoyama, R. "Power system restoration by joint usage of expert system and mathematical programing approach." IEEE Transactios on Power Systems. 10 (3). pp. 1473-1479. 1995. DOI: $10.1109 / 9780470545607 . \operatorname{ch} 70$

[3] Huang, Y. C. "Enhanced genetic algorithm- based fuzzy multi-objective approach to distribution network reconfiguration." IEE Proceedings Generation, Transmission and Distribution. 149 (5). pp. 615-620. 2002. DOI: 10.1049/ip-gtd:20020512

[4] Nagata, T., Sasaki, H. "A Multi-Agent Approach to Power System Restoration." IEEE Transactios on Power Systems. 17 (2). pp. 457-462. 2002. DOI: 10.1109/tpwrs.2002.1007918

[5] Nagata, T., Sasaki, H. "An efficient algorithm for distribution network restoration." In: Power Engineering Society Summer Meeting, 2001, Vol. 1. pp. 54-59, 15-19 July 2001. DOI: 10.1109/PESS.2001.969982

[6] Li, P., Song, B., Wang, W., Wang, T. "Multi-agent approach for service restoration of microgrid." In: Industrial Electronics and Applications (ICIEA), 2010 the 5th IEEE Conference on, pp. 962-966, 15-17 June 2010. DOI: 10.1109/ICIEA.2010.5515722

[7] Huang, M., Chen, C., Lin, C. "Innovation service restoration of distribution systems by considering short-term load forecasting of service zones." Electrical Power and Energy Systems. pp. 417-427. 2005. DOI: 10.1016/j.ijepes.2005.02.004 
[8] Li, H., Sun, H., Wen, J., He, S.C.H. "A Fully Decentralized Multi-Agent System for Intelligent Restoration of Power Distribution Network Incorporating Distributed Generations." IEEE Computational intelligence magazine. pp. 66-76. 2012. DOI: 10.1109/mci.2012.2215152

[9] Zidan, A., El-Saadany, E. F. "A Cooperative Multiagent Framework for Self-Healing Mechanisms in Distribution Systems." IEEE Transactions on Smart Grid. 3 (3). pp. 1525-1539. 2012. DOI: 10.1109/tsg.2012.2198247

[10] Kumar, Y., Das, B., Sharma, J. "Multiobjective, multicaonstraint service restoration of electric power distribution systems with priority customers." IEEE Transactions on Power Delivery. 23 (1). pp. 261-270. 2008. DOI: $10.1109 /$ tpwrd.2007.905412

[11] Ciric, R., Popovic, D. "Multi-objective distribution network restoration using heuristic approach and mix integer programming method." International Journal of Electrical Power \& Energy Systems. 22 (7). pp. 497-505. 2000. DOI: 10.1016/s0142-0615(00)00018-1

[12] Kersting, W. H. "Distribution System Modeling and Analysis." $2^{\text {nd }}$ ed., Boca Raton: CRC Press, 2007.

[13] Miu, K., Chiang, H., McNulty, R. "Multi-tier service restoration through network reconfiguration and capacitor control for large-scale radial distribution networks." IEEE Transactions on Power Systems. 15 (3). pp. 1001-1007. 2000. DOI: 10.1109/59.871725
[14] Weiss, G. "Multiagent Systems: A Modern Approach to Distributed Artificial Intelligence." Cambridge, MA: The MIT Press. 2000.

[15] The Foundation for Intelligent Physical Agents. [Online]. Available from: http://www.fipa.org [Accessed: February 2015].

[16] Kato, T., Kanamori, H., Suzuoki, Y., Funabashi, T. "Multi-agent based control and protection of power distributed system-Protection scheme with simplified information utilization-." In: Intelligent Systems Application to Power Systems, 2005. Proceedings of the 13th International Conference on, pp. 49-54, 6-10 Nov. 2005.

DOI: 10.1109/ISAP.2005.1599240

[17] Tsai, M., Pan, Y. "Application of BDI-based intelligent multi-agent systems for distribution system service restoration planning. European Transactions on Electrical Power. 21 (5). pp. 1783-1801. 2011.

DOI: 10.1002 etep.542

[18] Das, D. "Reconfiguration of distribution system using fuzzy multi-objective approach." International Journal of Electrical Power \& Energy Systems. 28 (5). pp. 331-338. 2006. DOI: 10.1016/j.ijepes.2005.08.018

[19] JADE agent development toolkit [Online]. Available from: http://jade. tilab.com [Accessed: $1^{\text {st }}$ February 2015]. 